\title{
Editorial: New Developments and Challenges in Advanced High-Strength Steels
}

\author{
Lijia Zhao ${ }^{1 *}$, John G. Speer ${ }^{2 *}$, Peter Hodgson ${ }^{3 *}$ and Nobuhiro Tsuji ${ }^{4,5 *}$ \\ ${ }^{1}$ Key Laboratory of Electromagnetic Processing of Materials (Ministry of Education), Northeastern University, Shenyang, China, \\ ${ }^{2}$ Advanced Steel Processing and Products Research Center, Colorado School of Mines, Golden, CO, United States, ${ }^{3}$ Institute for \\ Frontier Materials (IFM), Deakin University, Geelong, VIC, Australia, ${ }^{4}$ Department of Materials Science and Engineering, Kyoto \\ University, Kyoto, Japan, ${ }^{5}$ Elements Strategy Initiative for Structural Materials (ESISM), Kyoto University, Kyoto, Japan
}

Keywords: advanced high-strength steels, strength and ductility, automotive, hydrogen embrittlement, heat treatment

\section{Editorial on the Research Topic}

\section{New Developments and Challenges in Advanced High-Strength Steels}

Steel is a globally-used structural material and a major factor in advancing societies and economies. Advanced high-strength steels (AHSS) are a class of high-performance steels particularly important to the automotive industry due to the increased need for fuel efficiency, emissions reduction and passive safety. The research topic "New Developments and Challenges in Advanced High-Strength Steels" aims at collecting state-of-the-art research studies on design, processing and characterization of AHSS. This issue comprises seven peer-reviewed research articles covering a wide range of steel types like medium manganese (Mn) steel, twinning induced plasticity (TWIP) steel, transformation induced plasticity (TRIP) steel, quenched and partitioned (Q\&P) steel, low-carbon ferrite steel and press-hardened steel.

Among these studies, the influence of heat treatment routes on the microstructure and mechanical property of AHSS were extensively investigated and some new processing routes were proposed. Pan and $\mathrm{He}$ achieved three microstructural combinations of ferrite, austenite and/or martensite by varied heat treatment including intercritical annealing (IA), quenching and partitioning (Q\&P), and a combination of IA and Q\&P. The variation in volume fraction and stability of retained austenite was compared between those microstructures. Different combinations of high strength and high elongation were obtained by adjusting the processing route, illustrating how the tensile properties of medium $\mathrm{Mn}$ steel can be tuned to facilitate its applicability to a broad range of automotive needs. Glover et al. proposed novel processing routes to improve the mechanical properties of a medium $\mathrm{Mn}$ steel. The addition of either a tempering or austempering heat treatment to double soaking of a medium Mn steel was shown to increase the strength-ductility product as compared to a single intercritical annealing heat treatment. This work highlights additional opportunities to modify the mechanical properties of medium Mn steels. It is well-known that grain refinement can improve the strength of steels. Severe plastic deformation (SPD) processes are commonly employed to create UFG microstructures with average grain sizes smaller than $1 \mu \mathrm{m}$. However, there exists big difficulty in scaling up the SPD approaches for mass steel production. Park et al. conducted a novel cyclic heat treatment to generate UFG ferrite in a $2 \mathrm{Mn}-0.1 \mathrm{C}$ steel. The cyclic heat treatment was proved to be effective for decreasing the austenite grain size to $11 \mu \mathrm{m}$. A finegrained ferrite structure with a mean grain size of $4.5 \mu \mathrm{m}$ and nearly a random texture was successfully obtained only through the cyclic heat treatment and provided both high strength and large tensile ductility. 
As introduced above, grain refinement can result in additional strengthening, and can also lead to diverse tensile deformation features of AHSS. Kang et al. investigated the yielding behavior of a high Mn TWIP steel with a fine grain size cooled at three conditions including air cooling, furnace cooling and water quenching. Localized deformation was observed at the beginning of plastic deformation in the three specimens. As a result of the localized deformation and following a transition to uniform plastic deformation, a valley-type evolution of the strain hardening rate was observed at an early stage of the tensile deformation. This study helps understand the yielding and strain hardening behavior of fine-grained TWIP steel. As a traditional approach, predicting yielding behavior in forming operations is characterized using yield surfaces to describe material yielding in specific stress states. The prediction becomes complex and needs correction if the material has experienced previous straining. Thrun et al. examined the effects of unloading and subsequent reloading on yielding and tensile properties of a 304 stainless steel by interrupted uniaxial tensile testing. A yield point was introduced in the austenitic stainless steel. The results suggested that both strain aging and dislocation trapping mechanisms may be active in the matrix, which may require further consideration when forming austenitic stainless and new AHSS. Besides the cold-stamped AHSS introduced above, press-hardened steel (PHS) has played a critical role in crash intrusion resistance and weight reduction in recent decades. For PHS, especially when the tensile strength increases from 1,500-2,000 $\mathrm{MPa}$, hydrogen embrittlement (HE) is an inevitable challenge. Lin et al. investigated the role of vanadium carbide in $\mathrm{HE}$ for two PHS with $1,500 \mathrm{MPa}$ (22MnB5) and $2000 \mathrm{MPa}(34 \mathrm{MnB} 5 \mathrm{~V})$, respectively. It was found that the dispersed vanadium carbides (VCs) increased the strength and slightly the ductility of the $34 \mathrm{MnB} 5 \mathrm{~V}$ steel due to the synergetic effects of precipitation hardening and microstructural refinement. Microstructural refinement enhanced by VC improves the resistance to HE in $34 \mathrm{MnB5V}$ steel. It was found that the correlation between hydrogen trapping by $\mathrm{VC}$ and improvement of $\mathrm{HE}$ is not significant, presenting a challenge in designing irreversible trapping sites in future PHS.

In this collection of papers, observation of new phenomena became possible using advanced microstructural characterization techniques. Microstructural changes during intercritical annealing of a medium $\mathrm{Mn}$ steel were evaluated in situ with a high energy X-ray diffraction system by $\mathrm{Hu}$ et al. Observed changes in lattice parameters during isothermal holding were interpreted to reflect composition changes due to redistribution of the $\mathrm{C}$ and $\mathrm{Mn}$ between austenite and ferrite. In situ observation of phase transformation can provide a comprehensive understanding of microstructure responses during the heat treatment process of AHSS, guiding heat treatment design for optimal microstructure and properties.

We hope that the papers in the present Research Topic can stimulate new exciting research work in the continued development of AHSS.

\section{AUTHOR CONTRIBUTIONS}

All authors listed have made a substantial, direct, and intellectual contribution to the work, and approved it for publication.

\section{FUNDING}

This work was financially supported by the Elements Strategy Initiative for Structural Materials (ESISM; JPMXP0112101000), JST CREST (JPMJCR 1994), and KAKENHI (Grant-in-Aid for Scientific Research from the Japan Society for the Promotion of Science; 15H05767, 19H02459, and 20K14608), all from the Ministry of Education, Culture, Sports, Science and Technology (MEXT), Japan. The sponsors of the Advanced Steel Processing and Products Research Center (ASPPRC) at the Colorado School of Mines are gratefully acknowledged for their support. All the sources of support for the authors' efforts are gratefully acknowledged.

Conflict of Interest: The authors declare that the research was conducted in the absence of any commercial or financial relationships that could be construed as a potential conflict of interest.

Publisher's Note: All claims expressed in this article are solely those of the authors and do not necessarily represent those of their affiliated organizations, or those of the publisher, the editors and the reviewers. Any product that may be evaluated in this article, or claim that may be made by its manufacturer, is not guaranteed or endorsed by the publisher.

Copyright (C) 2021 Zhao, Speer, Hodgson and Tsuji. This is an open-access article distributed under the terms of the Creative Commons Attribution License (CC BY). The use, distribution or reproduction in other forums is permitted, provided the original author(s) and the copyright owner(s) are credited and that the original publication in this journal is cited, in accordance with accepted academic practice. No use, distribution or reproduction is permitted which does not comply with these terms. 